\title{
Actitudes hacia las matemáticas en estudiantes de Educación Primaria: Diferencias en función del curso y del género
}

\author{
Antonio Valle, Bibiana Regueiro, Isabel Piñeiro, Benigno Sánchez, \\ Carlos Freire y Mar Ferradás \\ Universidad de A Coruña (España)
}

\begin{abstract}
El principal objetivo de este trabajo es comprobar si hay diferencias en algunas variables vinculadas con las actitudes hacia las matemáticas en estudiantes de Educación Primaria en función del curso y del género. La muestra está integrada por 897 estudiantes de $5^{\circ}$ y $6^{\circ}$ curso de Educación Primaria (50.2\% chicos y $49.8 \%$ chicas). Los resultados de este trabajo indican que los chicos, en comparación con las chicas, tienen una competencia percibida más alta en matemáticas, están más motivados extrínseca e intrínsecamente y muestran unos niveles de ansiedad más bajos ante esta asignatura. En cuanto a las diferencias en función de la variable curso, los resultados indican que los estudiantes de $5^{\circ}$ curso tienen una competencia percibida más alta para las matemáticas, las perciben más útiles, están más motivados intrínsecamente hacia esta asignatura y también muestran unos niveles de ansiedad y unos sentimientos negativos hacia las matemáticas más bajos que los estudiantes de $6^{\circ}$ curso. Por tanto, las mujeres parecen mostrar un "perfil" de condiciones menos adaptativo que el de los hombres, tanto con respecto a su competencia percibida como a su motivación hacia las matemáticas y también en cuanto a las emociones asociadas a esta materia. En cuanto a las diferencias según el curso, los estudiantes de $5^{\circ}$ de Primaria son los que presentan unas condiciones actitudinales y motivacionales mucho más positivas que los estudiantes de $6^{\circ}$ curso.
\end{abstract}

Palabras clave: actitudes, matemáticas, género, curso, Educación Primaria.

Attitudes towards math in primary school students: Differences depending on the grade and gender. The main aim of this study is to check whether there are differences in some variables related to attitudes towards math in primary school students according to the course and gender. The sample consists of 897 students of the fifth and sixth year of primary education $(50.2 \%$ boys and $49.8 \%$ girls). The results indicate that the boys, compared to girls, have a higher perceived competence in math, they are more intrinsically motivated extrinsic and exhibit lower levels of anxiety. As for the differences in terms of this variable, the results indicate that students in grade 5 have a higher perceived competence for math, perceive most useful, are more intrinsically motivated to this subject and show anxiety levels and some negative feelings toward the lower than grade 6 . Therefore, girls show a "profile" of less adaptive than men conditions, both in terms of their perceived competition as their motivation towards math and also in terms of the emotions associated with this matter. As for the differences depending on the course, students from grade 5 are those with a much more positive attitudinal and motivational conditions than grade 6 .

Keywords: attitudes, math, gender, grade, primary education.

Correspondencia: Antonio Valle. Departamento de Psicología Evolutiva y de la Educación. Universidad de A Coruña. Campus de Elviña, s/n. C.P.: 15071. A Coruña (España). E-mail: vallar@udc.es 
La importancia de las actitudes y su papel fundamental en las matemáticas lleva siendo objeto de estudio e interés científico desde hace más de 50 años, siendo las diferencias de género y curso algunas de las cuestiones más debatidas (González-Pienda et al., 2012; Araújo, Aragón, Aguilar, Navarro, y Ruiz, 2014). Incluso, se ha destacado el papel motivacional que tienen las actitudes de los padres hacia los deberes escolares que realizan sus hijos en casa, especialmente en lo que se refiere al soporte afectivo y motivacional y no tanto en ayudar a resolver esos deberes (Regueiro et al., 2015).

Hace ya algunos años, Fennema y Sherman (1977, 1978) habían encontrado diferencias de género relacionadas con el éxito en las matemáticas. Al mismo tiempo, analizaron diversas variables afectivas y actitudinales encontrando una mayor percepción de utilidad y una mayor confianza en los hombres respecto a las mujeres. Estudios más actuales reflejan la idea por parte de ambos sexos, de que los chicos, más que las chicas, necesitan las matemáticas más para su vida adulta y para conseguir buenos trabajos. Además, ambos expresaron que a los chicos les gustan más las matemáticas y les resultan más fáciles, mientras las chicas las consideran más aburridas y difíciles (Brandell y Staberg, 2008). Al mismo tiempo, las chicas también se muestran menos seguras de sí mismas ante ellas (Frenzel, Pekrun, y Goetz, 2007).

Por su parte, Else-Quest, Hyde y Linn (2010) establecieron en este sentido que los chicos muestran mayor placer y orgullo, así como menor ansiedad y desesperanza ante las matemáticas que las chicas. Además, se sienten más motivados tanto extrínseca como intrínsecamente hacia esta materia. También respecto del género, otros autores (ver p.e., Fullarton, 1993; Thomas, 2000, Willis, 1995) encontraron una actitud más negativa hacia las matemáticas por parte de las mujeres, lo cual repercute, a su vez, en una baja implicación y menor efectividad en las mismas que los hombres. En lo que sí parecen coincidir la mayor parte de los estudios es que los chicos y las chicas presentan actitudes diferentes ante las matemáticas, estando los chicos más seguros sobre su competencia matemática (Preckel, Goetz, Pekrun, y Kleine, 2008).

Sin embargo, algunos estudios (p.e., Carrel, Page, y West, 2009; Dee, 2007) muestran que el hecho de tener una profesora de matemáticas mejora el desempeño de las chicas en esta materia. Niederle y Vesterlund (2009) establecen que el número de profesores varones en matemáticas es mayor al de mujeres, lo cual refuerza ciertos estereotipos destacados aquí.

Otros estudios no encuentran tan claramente el área de matemáticas como un dominio masculino. Así, Forgasz (2000) en una extensa revisión para intentar contrastar la hipótesis del dominio masculino en las matemáticas, en una muestra de estudiantes australianos, reveló que los chicos consideraban las matemáticas más difíciles que las chicas, necesitando apoyo adicional. Además, destacaba el mayor interés y aprecio de las matemáticas por parte de las chicas. 
Kloosterman, Tassell, Ponniah, y Essex (2001), en una investigación sobre estudiantes de secundaria y universidad, concluyeron que la percepción de los estudiantes es que las matemáticas no eran una cuestión de género y atribuían un carácter neutral a las mismas (en el que el género no se tenía en cuenta). Se veía un aprecio por igual hacia las matemáticas en alumnos y alumnas. También, Hanna (2003) realizó una revisión sobre la equidad del género en las matemáticas, examinando los tres estudios de la Internacional Association for the Evaluation of Educational Achievement (IEA): First, Second and Third Internacional Mathematics Science Study (FIMMS, SIMMS y TIMMS, respectivamente). Aquí se vio una gran variabilidad de un país a otro respecto a las diferencias de género. La igualdad de género se observa en el grupo de alumnos y alumnas de 13 años de edad. En el grupo del alumnado de 17 años de edad, los chicos presentan mejores resultados que las chicas en algunas áreas matemáticas. Ercikan McCreith, y Lapointe (2005), tomando como referencia el TIMMS, encontraron diferencias significativas en relación al género del alumnado y su éxito en las matemáticas y corroboraron las notables diferencias entre países. También afirmaron que en la escuela elemental y media no se encuentran diferencias de género respecto al éxito en matemáticas. Niederle y Vesterlund (2009) han encontrado una ligera diferencia entre hombres y mujeres respecto al rendimiento en matemáticas en el nivel de educación secundaria que cada día se tiende a igualar.

González-Pienda et al. (2012), en una investigación sobre alumnado de primaria y secundaria de alumnos españoles y brasileños, encuentran diferencias de género sobre las dimensiones del Inventario de Actitudes hacia las Matemáticas (IAM), pero recalcan que tales efectos están mediatizados poderosamente por la variable curso, siendo la interacción entre las variables curso y género muy significativa. Concluyen así, que la incidencia del género tiene una magnitud pequeña, aun siendo significativa, en el rendimiento en matemáticas, no pudiendo hablarse de una incidencia de esta variable en la actitud hacia las matemáticas.

También en la literatura científica se percibe una gran influencia de la variable curso en las actitudes hacia las matemáticas. González-Pienda et al. (2006) establecen que a medida que el estudiante avanza en la escolaridad obligatoria su actitud hacia las matemáticas se va haciendo más negativa. Watt (2000) llevó a cabo un estudio donde pretendía conocer la relación entre las actitudes y el rendimiento académico en el área de matemáticas, a medida que el sujeto progresaba en su escolaridad y teniendo en cuenta los distintos cursos. Los resultados mostraron un cambio de actitudes hacia las matemáticas a medida que el sujeto avanzaba en su escolaridad, manifestando actitudes más negativas hacia el aprendizaje de las matemáticas, así como una tendencia al dominio masculino en esta área.

Utsumi y Mendes (2000) destacaron que cuando el alumno pasa de la enseñanza básica a la enseñanza media, sus actitudes hacia las matemáticas se suelen volver más 
negativas. Así, de los datos recogidos, comprobaron que la actitud hacia las matemáticas empeoraba en el alumnado de 11 y 12 años respecto al de cursos anteriores y el alumnado con 16 años tenía una concepción aún más negativa del aprendizaje matemático. Warrington, Younger, y Williams (2000) encuentran en su investigación que a medida que los estudiantes avanzan de curso las actitudes hacia las matemáticas por parte de los alumnos van siendo más negativas.

En base a estos planteamientos, el principal objetivo de este trabajo es comprobar si hay diferencias estadísticamente significativas en algunas variables vinculadas con las actitudes hacia las matemáticas en estudiantes de Educación Primaria en función del curso y del género.

\section{MÉTODO}

\section{Participantes}

La muestra está integrada por 897 estudiantes pertenecientes a 13 centros públicos de Educación Primaria de la provincia de A Coruña (España). El 50.2\% son hombres y el $49.8 \%$ son mujeres, de edades comprendidas entre los 9 y los 13 años $(M=10.77)$. Del total, 437 de ellos cursaban $5^{\circ}$ curso de Educación Primaria (223 alumnos y 213 alumnas) y 460 cursaban $6^{\circ}$ curso de Educación Primaria (227 alumnos y 233 alumnas).

\section{Instrumentos}

Para medir las actitudes del alumnado hacia las matemáticas se ha utilizado el IAM (Inventario de Actitudes hacia las Matemáticas). Este instrumento resulta de la ampliación de la Fennema-Sherman Mathematics Attitudes Scales (FSS) de Fennema y Sherman (1976). Se trata de una versión ampliada de la escala con algunas modificaciones y que ha sido adaptada al español incorporando nuevas dimensiones destinadas a medir de un modo más preciso las actitudes y la motivación del alumnado hacia las matemáticas (Cueli et al., 2014; González-Pienda et al., 2012; Silva, 2005). En este caso se utilizaron las siguientes dimensiones del IAM.

- Competencia percibida para las matemáticas $(\alpha=.75)$ : evalúa el grado de confianza en uno mismo para aprender y obtener buenos resultados en matemáticas.

- Ansiedad ante las matemáticas $(\alpha=.77)$ : evalúa el grado de ansiedad del alumno ante las matemáticas.

- Percepción de utilidad de las matemáticas $(\alpha=.60)$ : evalúa el grado en que los estudiantes perciben la utilidad de los aprendizajes realizados en matemáticas, especialmente de cara al futuro.

- Motivación de logro hacia las matemáticas $(\alpha=.78)$ : evalúa la motivación orientada a conseguir las mejores notas y ser el mejor en matemáticas. 
- Motivación intrínseca hacia las matemáticas $(\alpha=.72)$ : evalúa la motivación orientada hacia el aprendizaje y la comprensión de los contenidos matemáticos por el placer y satisfacción personal y desinteresada que conlleva el trabajo en este tipo de contenidos.

- Sentimientos negativos provocados por las matemáticas $(\alpha=.70)$ : evalúa la presencia e intensidad de sentimientos negativos generados por el trabajo en el área de matemáticas.

Los ítems de cada una de las dimensiones tienen un formato tipo likert con cinco alternativas de respuesta que van desde 1 (totalmente falso) hasta 5 (totalmente cierto).

\section{Procedimiento}

Los datos fueron recogidos durante el horario escolar por personal externo al propio centro educativo, previo consentimiento del equipo directivo y de los profesores de los alumnos. Antes de la recogida de datos, realizada en un único momento temporal, se informó a los participantes de la importancia de responder sinceramente a las distintas preguntas planteadas, insistiendo en su carácter totalmente confidencial.

\section{Análisis de datos}

Se llevó a cabo un Análisis Multivariado de Varianza (MANOVA) con el fin de comprobar si hay diferencias estadísticamente significativas en las variables vinculadas con la actitud hacia las matemáticas (variables dependientes) en función del curso y del género (variables independientes). Para la interpretación de los tamaños del efecto se utilizó el criterio establecido por Cohen (1988), según el cual, un efecto es pequeño cuando $\eta_{\mathrm{p}}{ }^{2}=.01(d=.20)$, el efecto es medio cuando $\eta_{\mathrm{p}}{ }^{2}=.059(d=.50)$ y el tamaño del efecto es grande si $\eta_{\mathrm{p}}{ }^{2}=.138(d=.80)$.

\section{RESULTADOS}

A nivel multivariado, los resultados indican que hay diferencias estadísticamente significativas en las seis variables vinculadas con las actitudes hacia las matemáticas, tomadas conjuntamente, en función del curso $\left(\lambda_{\text {Wilks }}=.953\right.$; $\left.F(6,888)=7.29 ; p<.001 ; \eta_{\mathrm{p}}{ }^{2}=.047\right)$ y del género $\left(\lambda_{\text {Wilks }}=.955 ; F(6,888)=7.05 ; p<.001\right.$; $\left.\eta_{\mathrm{p}}{ }^{2}=.045\right)$. Sin embargo, la interacción entre el género y el curso no es estadísticamente significativa $\left(\lambda_{\text {Wilks }}=.994 ; F(6,888)=0.94 ; p=.463 ; \eta_{\mathrm{p}}{ }^{2}=.006\right)$. En ambos casos, los tamaños del efecto son pequeños.

En cuanto al efecto género, los análisis univariados indican que hay diferencias estadísticamente significativas entre hombres y mujeres en la competencia percibida en matemáticas $\left(F(1,893)=10.87 ; p<.001 ; \eta_{\mathrm{p}}{ }^{2}=.012\right)$, en ansiedad ante las matemáticas 
$\left(F(1,893)=10.57 ; p<.001 ; \eta_{\mathrm{p}}^{2}=.012\right)$, en la motivación de logro en matemáticas $\left(F(1,893)=16.47 ; p<.001 ; \eta_{\mathrm{p}}{ }^{2}=.018\right)$ y en la motivación intrínseca en matemáticas $\left(F(1,893)=6.05 ; p<.01 ; \eta_{\mathrm{p}}{ }^{2}=.007\right)$. No se observan diferencias significativas en función del género en la percepción de utilidad de las matemáticas $(F(1,893)=0.01 ; p=.934$; $\left.\eta_{\mathrm{p}}{ }^{2}=.000\right)$ ni en los sentimientos negativos provocados por las matemáticas $\left(F(1,893)=1.47 ; p=.226 ; \eta_{\mathrm{p}}{ }^{2}=.002\right)$. En todos los casos, los tamaños del efecto son pequeños.

Tabla 1. Estadísticos descriptivos (media, desviación típica) correspondientes a las variables vinculadas con las actitudes hacia las matemáticas en función del curso y del género

\begin{tabular}{|c|c|c|c|c|c|c|}
\hline & \multicolumn{2}{|c|}{$5^{\circ}$ Primaria } & \multicolumn{2}{|c|}{$6^{\circ}$ Primaria } & \multicolumn{2}{|c|}{ Total } \\
\hline & $M$ & $D T$ & $M$ & $D T$ & $M$ & $D T$ \\
\hline \multicolumn{7}{|c|}{ Competencia percibida matemáticas } \\
\hline Hombres & 4.22 & 0.75 & 4.03 & 0.79 & 4.12 & 0.78 \\
\hline Mujeres & 4.08 & 0.69 & 3.84 & 0.73 & 3.95 & 0.72 \\
\hline Total & 4.15 & 0.73 & 3.93 & 0.77 & 4.04 & 0.75 \\
\hline \multicolumn{7}{|c|}{ Ansiedad ante las matemáticas } \\
\hline Hombres & 1.82 & 0.98 & 2.14 & 1.09 & 1.94 & 1.05 \\
\hline Mujeres & 1.97 & 1.01 & 2.44 & 1.10 & 2.22 & 1.08 \\
\hline Total & 1.89 & 0.99 & 2.29 & 1.07 & 2.10 & 1.07 \\
\hline \multicolumn{7}{|c|}{ Percepción utilidad de las matemáticas } \\
\hline Hombres & 4.61 & 0.65 & 4.49 & 0.80 & 4.55 & 0.73 \\
\hline Mujeres & 4.59 & 0.71 & 4.50 & 0.68 & 4.54 & 0.70 \\
\hline Total & 4.60 & 0.68 & 4.50 & 0.74 & 4.55 & 0.71 \\
\hline \multicolumn{7}{|c|}{ Motivación de logro matemáticas } \\
\hline Hombres & 4.14 & 0.92 & 3.97 & 1.16 & 4.06 & 1.05 \\
\hline Mujeres & 3.83 & 1.05 & 3.72 & 1.09 & 3.78 & 1.07 \\
\hline Total & 3.99 & 0.99 & 3.85 & 1.13 & 3.92 & 1.07 \\
\hline \multicolumn{7}{|c|}{ Motivación intrínseca matemáticas } \\
\hline Hombres & 3.89 & 0.82 & 3.68 & 0.88 & 3.78 & 0.86 \\
\hline Mujeres & 3.85 & 0.78 & 3.44 & 0.87 & 3.64 & 0.85 \\
\hline Total & 3.87 & 0.80 & 3.56 & 0.88 & 3.71 & 0.86 \\
\hline \multicolumn{7}{|c|}{ Sentimientos negativos matemáticas } \\
\hline Hombres & 1.74 & 0.88 & 1.87 & 0.95 & 1.80 & 0.91 \\
\hline Mujeres & 1.61 & 0.78 & 1.85 & 0.84 & 1.74 & 0.82 \\
\hline Total & 1.68 & 0.83 & 1.86 & 0.89 & 1.77 & 0.87 \\
\hline
\end{tabular}

En cuanto al efecto del curso, los resultados muestran que hay diferencias estadísticamente significativas entre estudiantes de $5^{\circ}$ y $6^{\circ}$ de Primaria en competencia percibida en matemáticas $\left(F(1,893)=18.84 ; p<.001 ; \eta_{\mathrm{p}}{ }^{2}=.021\right)$, en ansiedad ante las matemáticas $\left(F(1,893)=31.11 ; p<.001 ; \eta_{\mathrm{p}}{ }^{2}=.034\right)$, en la percepción de utilidad de las matemáticas $\left(F(1,893)=4.34 ; p<.05 ; \eta_{\mathrm{p}}{ }^{2}=.005\right)$, en motivación intrínseca en matemáticas $\left(F(1,893)=29.06 ; p<.001 ; \eta_{\mathrm{p}}{ }^{2}=.032\right)$ y también en los sentimientos negativos provocados por las matemáticas $\left(F(1,893)=10.50 ; p<.001 ; \eta_{\mathrm{p}}{ }^{2}=.012\right)$. No se aprecian diferencias significativas entre cursos en la motivación de logro en matemáticas $(F(1,893)=3.49$; $\left.p=.062 ; \eta_{\mathrm{p}}{ }^{2}=.004\right)$. También en estos casos, los tamaños del efecto son pequeños. 
Si tenemos en cuenta las medias de ambos grupos (ver tabla 1) en las variables con resultados significativos, se puede observar que los hombres, en comparación con las mujeres, tienen una competencia percibida más alta en matemáticas, están más motivados extrínseca e intrínsecamente y muestran unos niveles de ansiedad más bajos ante esta asignatura (ver figuras 1,4 y 5).

Por lo que respecta a las diferencias entre cursos, teniendo en cuenta las medias de los dos grupos (ver tabla 1), los estudiantes de $5^{\circ}$ curso tienen una competencia percibida para las matemáticas más alta, las perciben más útiles, están más motivados intrínsecamente hacia esta asignatura y también muestran unos niveles de ansiedad y unos sentimientos negativos hacia las matemáticas más bajos que los estudiantes de $6^{\circ}$ curso (ver figuras $1,3,5,2$ y 6 ).

Figura 1. Valores medios en la variable Competencia percibida en matemáticas en función del curso y del género

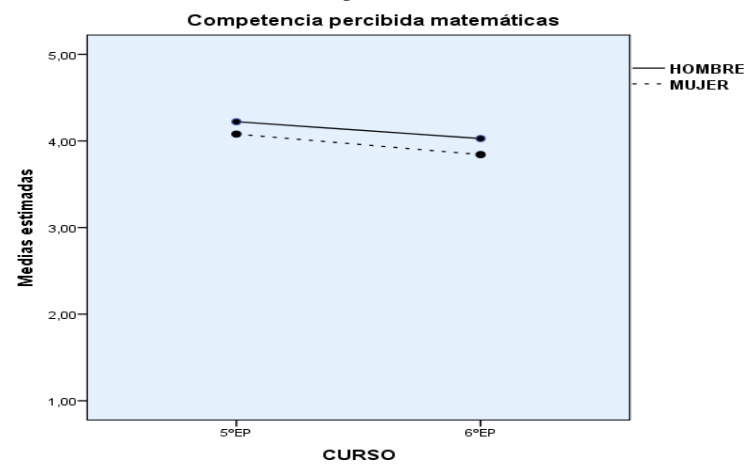

Figura 2. Valores medios en la variable Ansiedad ante las matemáticas en función del curso y del género

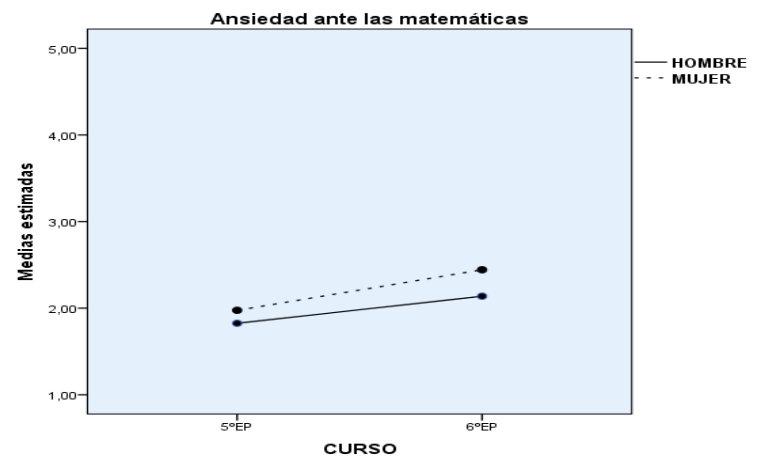


Teniendo en cuenta cada una de las variables dependientes, la interacción entre género y curso tampoco es estadísticamente significativa en la competencia percibida en matemáticas (ver figura 1) $\left(F(1,893)=0.18 ; p=.668 ; \eta_{\mathrm{p}}{ }^{2}=.000\right)$; en la ansiedad ante las matemáticas (ver figura 2$)\left(F(1,893)=1.23 ; p=.267 ; \eta_{\mathrm{p}}{ }^{2}=.001\right)$; en la percepción de utilidad de las matemáticas (ver figura 3$)\left(F(1,893)=0.10 ; p=.755 ; \eta_{\mathrm{p}}{ }^{2}=.000\right)$; en la motivación de logro en matemáticas (ver figura 4$)\left(F(1,893)=0.13 ; p=.715 ; \eta_{\mathrm{p}}{ }^{2}=.000\right)$; en la motivación intrínseca en matemáticas (ver figura 5) $\left(F(1,893)=3.15 ; p=.076 ; \eta_{\mathrm{p}}{ }^{2}=.004\right)$; ni en los sentimientos negativos ante las matemáticas (ver figura 6) $(F(1,893)=0.95 ; p=.330$; $\left.\eta_{\mathrm{p}}^{2}=.001\right)$.

Figura 3. Valores medios en la variable Percepción de utilidad de las matemáticas en función del curso y del género

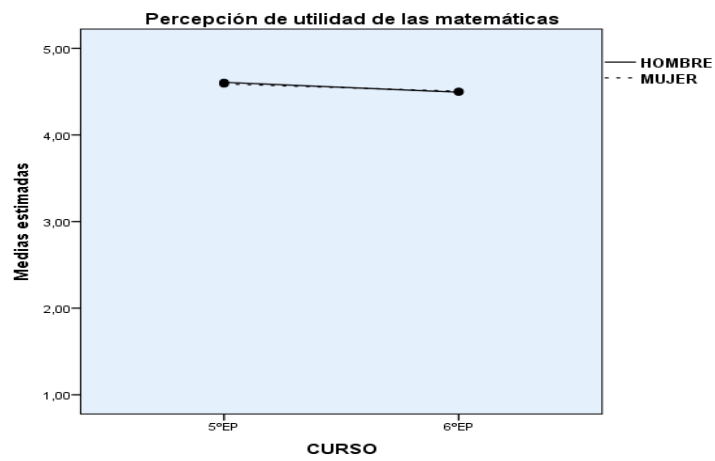

Figura 4. Valores medios en la variable Motivación de logro en matemáticas en función del curso y del género

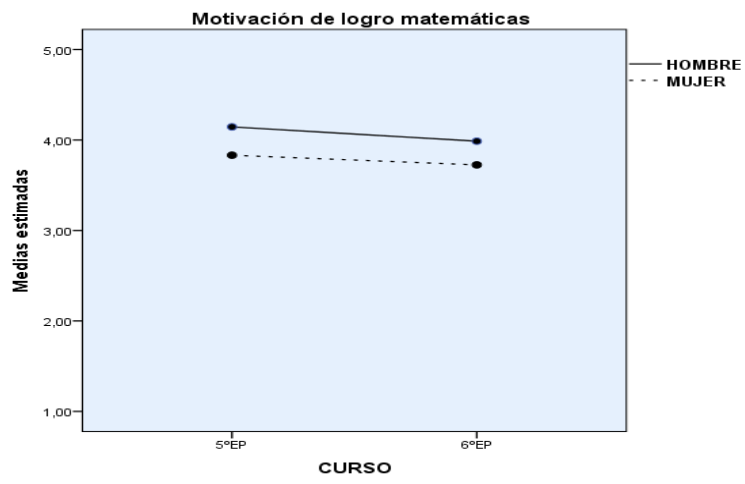


Figura 5. Valores medios en la variable Motivación intrínseca en matemáticas en función del curso y del género

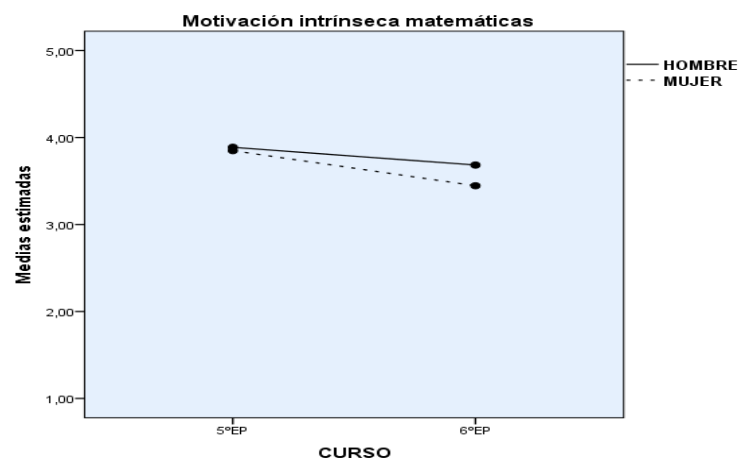

Figura 6. Valores medios en la variable Sentimientos negativos provocados por las matemáticas en función del curso y del género

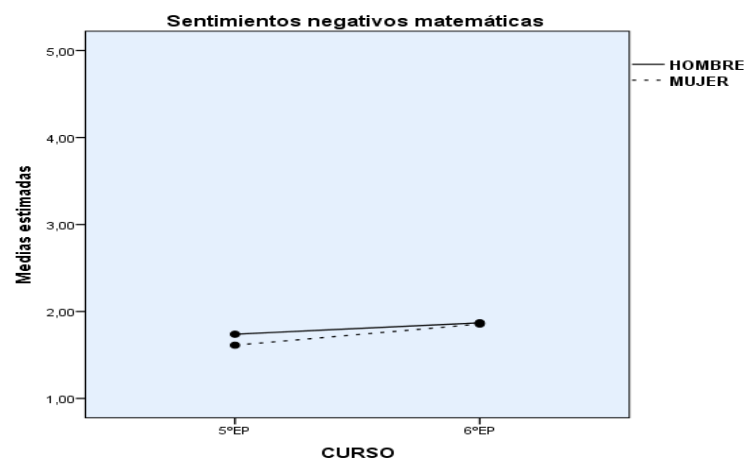

\section{DISCUSIÓN Y CONCLUSIONES}

Los resultados de este trabajo indican que los chicos, en comparación con las chicas, tienen una competencia percibida más alta en matemáticas, están más motivados extrínseca e intrínsecamente y muestran unos niveles de ansiedad más bajos ante esta asignatura. En algunos estudios realizados hace años (ver p.e., Fennema y Sherman, 1977, 1978) ya se encontraron evidencias de la existencia de diferencias de género en relación con las matemáticas. Según estos trabajos, los hombres mostraban más confianza hacia las matemáticas que las mujeres y consideraban, además, que tenían más utilidad para ellos que para ellas. En otros estudios posteriores (ver p.e., Watt, 2000) también se confirma que las mujeres se perciben menos competentes para las matemáticas que los hombres. 
Algunos de los resultados de otros estudios más recientes (p.e., González-Pienda et al., 2012) también ponen de manifiesto que las mujeres se perciben con más bajos niveles de competencia para las matemáticas que los hombres. Las mujeres suelen considerar las matemáticas aburridas y difíciles en mayor medida que los hombres (Brandell y Staberg, 2008) y se muestran menos seguras de sí mismas en la materia (Frenzel et al., 2007). Los hombres, por su parte, muestran más placer y orgullo en relación con las matemáticas, así como menor ansiedad y desesperanza ante las mismas. Además, se sienten más motivados tanto extrínseca como intrínsecamente para enfrentarse a dicha asignatura (Else-Quest et al., 2010). Los hombres son más seguros de sí mismos que las mujeres en lo que respecta a la competencia matemática (Preckel et al., 2008). Este estereotipo quizás se ve reforzado, en parte, porque la proporción de profesores varones de matemáticas es mayor que en otras asignaturas (Niederle y Vesterlund, 2009). De hecho, se ha comprobado en algunos estudios (ver p.e., Carrell et al., 2009; Dee, 2007) que el tener una profesora de matemáticas o de ciencias, mejora el desempeño de las mujeres en estas asignaturas.

De todos modos, también hay otros trabajos (p.e., Forgasz, 2000) que ponen de manifiesto que los hombres consideran las matemáticas más difíciles que las mujeres, necesitando incluso de ayuda adicional para esta asignatura. En este mismo trabajo, también se comprobó que las mujeres se interesaban y apreciaban más las matemáticas que los hombres.

En cuanto a las diferencias en función de la variable curso, los resultados indican que los estudiantes de $5^{\circ}$ curso tienen una competencia percibida más alta para las matemáticas, las perciben más útiles, están más motivados intrínsecamente hacia esta asignatura y también muestran unos niveles de ansiedad y unos sentimientos negativos hacia las mismas más bajos que los estudiantes de $6^{\circ}$ curso.

Estos resultados demuestran que a medida que se avanza en la escolaridad se observan unas actitudes más negativas hacia el aprendizaje de contenidos matemáticos (Núñez et al., 2005; González-Pienda et al., 2006; Utsumi y Mendes, 2000; Warrington et al., 2000). Probablemente, al disminuir la confianza en las propias capacidades para las matemáticas en $6^{\circ}$ curso, ello provoca una disminución del interés por esta asignatura despertando sentimientos y emociones negativas y aumentando significativamente el grado de ansiedad ante los aprendizajes realizados en esta materia.

Por tanto, después de analizar las diferencias de género en algunas variables más directamente relacionadas con las actitudes hacia las matemáticas, los resultados de este trabajo indican que las mujeres parecen mostrar un "perfil" de condiciones menos adaptativo que el de los hombres, tanto con respecto a su competencia percibida como a su motivación hacia las matemáticas, y también en cuanto a las emociones asociadas a esta materia. Posiblemente, los sentimientos negativos asociados a las matemáticas y la baja competencia percibida en esa materia están relacionados entre sí, ya que todo parece 
indicar que es más probable que un evento sea interpretado como una amenaza o que provoque sentimientos negativos en una persona, cuando esa persona tiene un bajo sentido de eficacia para enfrentarse a dicho evento (Nie, Lau, y Liau, 2011). De esto se deduce que las creencias de autoeficacia son un pilar fundamental desde el punto de vista motivacional (Valle, Regueiro, Rodríguez, Piñeiro, Freire et al., 2015).

Asimismo, se han encontrado diferencias significativas en función del curso en muchas de las variables estudiadas. En este caso, son los estudiantes de $5^{\circ}$ curso de Primaria los que presentan unas condiciones actitudinales y motivacionales mucho más positivas que los estudiantes de $6^{\circ}$ curso. Este resultado es coincidente en algunos aspectos con otros trabajos que indican que a medida que los estudiantes avanzan de curso disminuye su motivación intrínseca, su interés, su percepción de utilidad, el aprovechamiento del tiempo y su actitud hacia los deberes (ver p.e., Regueiro, Suárez, Valle, Núñez, y Rosário, 2015; Valle, Regueiro, Estévez et al., 2015; Valle, Regueiro, Rodríguez, Piñeiro, Ferradás et al., 2015).

De los resultados de este trabajo se pueden extraer algunas reflexiones importantes de cara a los procesos de enseñanza y aprendizaje de las matemáticas, especialmente en lo que se refiere al género y al curso. Aunque muchos de los resultados reflejan diferencias estadísticamente significativas, hay que tener en cuenta que a nivel práctico la magnitud es más bien pequeña, tal y como se puede ver en los tamaños del efecto encontrados. Esto es sumamente importante, ya que permite conocer la relevancia práctica de las diferencias estadísticamente significativas que reflejan los propios resultados.

Por lo que se refiere a las diferencias de género en algunas variables afectivomotivacionales, que coinciden en general con los resultados de otros trabajos ya mencionados, todo parece indicar que las líneas de actuación deben ir encaminadas, por un lado, a sensibilizar al profesorado de que las matemáticas no son algo exclusivamente de hombres y, por otro, a modificar las bajas creencias de autoeficacia ante las matemáticas que parecen tener muchas mujeres. Este cambio en las creencias de autoeficacia sólo se va a producir después de experimentar el éxito en matemáticas. Por eso, el papel del profesorado es fundamental a la hora de diseñar y prescribir tareas y actividades matemáticas que estén adaptadas a la diversidad de dificultades, necesidades y perfiles motivacionales de los estudiantes.

Sin embargo, si tenemos en cuenta todas estas variables según se avanza de curso, los resultados no parecen invitar al optimismo. Parece un tanto paradójico que al mismo tiempo que los estudiantes avanzan de curso y adquieren más conocimientos y habilidades matemáticas, también se sientan menos competentes para las matemáticas, se encuentren menos motivados y muestren unos niveles de ansiedad y unos sentimientos más negativos hacia esta materia. Desde una perspectiva educativa, esto resulta a todas luces un fracaso en la enseñanza y el aprendizaje de las matemáticas. Es inverosímil pensar 
que en otros procesos de aprendizaje pueda acontecer algo parecido, es decir, que cuanto más aprende una persona y cuantas más competencias adquiere, también se siente menos competente, con mayor ansiedad y con unos sentimientos más negativos hacia dichos aprendizajes. ¿Alguien se puede imaginar otros aprendizajes donde a medida que uno va adquiriendo más habilidades y conocimientos, también se va sintiendo menos capaz y menos competente?

Desde un punto de vista curricular, dado que los contenidos matemáticos van incrementando progresivamente su dificultad, es fácil que un alumno que ya tiene dificultades tempranamente, cada vez encuentre más dificultad para afrontar esos aprendizajes. Pero como en el contexto del aula es habitual la comparación entre compañeros, la autoestima y la autoeficacia de esos alumnos con dificultades decrece, y también su motivación e interés. Por eso, sobre todo en matemáticas, es muy importante que se actúe nada más se perciba que el alumno tiene alguna dificultad, pues si esto no se hace ocurrirá, inevitablemente, que a medida que avanza de curso sus posibilidades en matemáticas disminuyen. Por eso, en matemáticas se necesita claramente: (a) una identificación temprana de la dificultad, (b) la ayuda rápida para que el alumno no pierda confianza en sí mismo en esta área, y (c) eliminar en lo posible la comparación social y tratar de promover la percepción de mejora personal.

En futuras investigaciones será necesario analizar con mayor detalle estos resultados a través de diseños más potentes, con el fin de averiguar si, efectivamente, hay esas diferencias entre chicos y chicas en las actitudes hacia las matemáticas y, al mismo tiempo, comprobar si esa disminución de las condiciones que garantizan un aprendizaje de calidad continúa produciéndose a medida que los estudiantes avanzan de curso.

\section{Agradecimientos}

Este trabajo se ha desarrollado gracias a la financiación del proyecto de investigación EDU2013-44062-P, perteneciente al Plan Estatal de Investigación Científica y Técnica y de Innovación 2013-2016 (MINECO) y al financiamiento recibido por una de las autoras en el Programa FPU del Ministerio de Educación, Cultura y Deporte.

\section{REFERENCIAS}

Araújo, A., Aragón, E., Aguilar, M., Navarro, J.I., y Ruiz, G. (2014). Un estudio exploratorio para la adaptación de la versión española revisada del "Early Numeracy Test-R" para evaluar el aprendizaje matemático temprano. European Journal of Education and Psychology, 7(2), 83-93.

Brandell, G., y Staberg, E.M. (2008). Mathematics: A female, male or gender-neutral domain? A study of attitudes among students at secondary level. Gender and Education, 20, 495-509.

Carrell, S.E., Page, E., y West, J.E. (2009). Sex and science: How professor gender perpetuates the gender gap. The Quarterly Journal of Economics, 125, 1101-1114. 
Cohen, J. (1988). Statistical power analysis for the behavioral sciences. New Jersey: Lawrence Erlbaum.

Cueli, M., González-Castro, P., Álvarez, L., García, T., y González-Pienda, J.A. (2014). Variables afectivo-motivacionales y rendimiento en matemáticas: Un análisis bidireccional. Revista Mexicana de Psicología, 31(2), 153-163.

Dee, T.S. (2007). Teachers and the gender gaps in student achievement. Journal of Human Resources, 42(3), 528-554.

Else-Quest, N.M., Hyde, J.S., y Linn, M. (2010). Cross-national patterns of gender differences in mathematics: A meta-analysis. Psychological Bulletin, 136(1), 103-127.

Ercikan, K., McCreith, T., y Lapointe, V. (2005). Factors associated with mathematics achievement and participation in advanced mathematics courses: an examination of gender differences from an internacional perspective. School Science and Mathematics, 105(1), 5-10.

Fennema, E., y Sherman, J.A. (1976). Fennema-Sherman Mathematics Attitudes Scales: Instruments designed to measure attitudes toward the learning of mathematics by females and males. Journal for Research in Mathematics Education, 7(5), 324-326.

Fennema, E., y Sherman, J.A. (1977). Sex-related differences in mathematics achievement, spatial visualization and affective factors. American Educational Research Journal, 14(1), 51-71.

Fennema, E., y Sherman, J.A. (1978). Sex-related differences in mathematics achievement and related factors: A further study. Journal for Research in Mathematics Education, 9(3), 189203.

Forgasz, H.J. (2000). The gender-stereotyping of mathematics: Pre-service teachers' views. Comunicación presentada en The Conference of the Australian Association for Research in Education [AARE]. Sydney, 4-7 de Diciembre.

Frenzel, A.C., Pekrun, R., y Goetz, T. (2007). Girls and mathematics - A "hopeless" issue? A controlvalue approach to gender differences in emotions towards mathematics. European Journal of Psychology of Education, 22(4), 497-514.

Fullarton, S. (1993). Confidence in mathematics: the effects of gender. Geelong: Deakin University Press.

González-Pienda, J.A., Fernández-Cueli, M., García, T., Suárez, N., Fernández, E., Tuero-Herrero, E., y Helena da Silva, E. (2012). Diferencias de género en actitudes hacia las matemáticas en la enseñanza obligatoria. Revista Iberoamericana de Psicología y Salud, 3(1), 55-73.

Gonzalez-Pienda, J.A., Núñez, J.C., Solano, P., da Silva, E.H., Rosario, P., Mourâo, R., y Valle, A. (2006). Olhares de género face á matemática: uma investigaçao no ensino obrigatório español. Estudos de Psicología, 11(2), 135-141.

Hanna, G. (2003). Reaching gender equity in Mathematics education. The Educational Forum, 67(3), 204-214.

Kloosterman, P., Tassell, J.H., Ponniah, A.G., y Essex, N.K. (2001). Mathematics as a gendered domain in the United States. The American Educational Research Association, 13, 1-15.

Nie, Y, Lau, S., y Liau, A.K. (2011). Role of academic self-efficacy in moderating the relation between task importance and test anxiety. Learning and Individual Differences, 21, 736741

Niederle, M., y Vesterlund, L. (2009). Explaining the gender gap in math test scores: The role of competition. The Journal of Economic Perspectives, 24(2), 129-144.

Núñez, J.C., González-Pienda, J.A., Álvarez, L., González-Castro, P., González-Pumariega, S., Roces, C., Castejón, L., Bernardo, A., Solano, P., García, D., Silva, E. H., Rosário, P., y Rodrigues, L.S. (2005). Las actitudes hacia las matemáticas: Perspectiva evolutiva. En Actas do VIII Congreso Galaico-Portugués de Psicopedagoxía (pp. 2389-2396). Braga: Universidade do Minho. 
Preckel, F., Goetz, T., Pekrun, R., y Kleine, M. (2008). Gender differences in gifted and averageability students: Comparing girls' and boys' achievement, self-concept, interest, and motivation in mathematics. Gifted Child Quarterly, 52, 146-59.

Regueiro, B., Rodríguez, S., Piñeiro, I., Estévez, I., Ferradás, M., y Suárez, N. (2015). Diferencias en la percepción de la implicación parental en los deberes escolares en función del nivel de motivación de los estudiantes. European Journal of Investigation in Health, Psychology and Education, 5(3), 313-323.

Regueiro, B., Suárez, N., Valle, A., Núñez, J.C., y Rosário, P. (2015). La motivación e implicación en los deberes escolares a lo largo de la escolaridad obligatoria. Revista de Psicodidáctica, 20(1), 47-63.

Silva, E.H. (2005). Actitudes hacia el aprendizaje de las matemáticas (Tesis Doctoral no publicada). Departamento de Psicología, Universidad de Oviedo, Oviedo.

Thomas, J.P. (2000). Influences on mathematics learning and attitudes among African American high school students. The Journal of Negro Education, 69(3), 165-183.

Utsumi, M.C., y Mendes, C.R. (2000). Researching the attitudes towards mathematics in basic education. Educational Psychology, 20(2), 237-243.

Valle, A., Regueiro, B., Estévez, I., Piñeiro, I., Rodríguez, S., y Freire, C. (2015). Implicación y motivación hacia los deberes escolares en los estudiantes de Primaria según el rendimiento académico y el curso. European Journal of Investigation in Health, Psychology and Education, 5(3), 345-355.

Valle, A., Regueiro, B., Rodríguez, S., Piñeiro, I., Ferradás, M., y Freire, C. (2015). ¿Es diferente la implicación en los deberes escolares según el rendimiento académico de los estudiantes? Revista de Estudios e Investigación en Psicología y Educación, 2(2), 12-17.

Valle, A., Regueiro, B., Rodríguez, S., Piñeiro, I., Freire, C., Ferradás, M., y Suárez, N. (2015). Perfiles motivacionales como combinación de expectativas de autoeficacia y metas académicas en estudiantes universitarios. European Journal of Education and Psychology, $8(1), 1-8$.

Warrington, M., Younger, M., y Williams, J. (2000). Student attitudes, image and the gender gap. British Educational Research Journal, 26(3), 393-407.

Watt, H.M.G. (2000). Measuring attitudinal change in mathematics and English over first year of junior high school: A multidimensional analysis. The Journal of Experimental Education, 68(4), 331-361.

Willis, S. (1995). Gender reform through school mathematics in equity mathematics education. En P. Rogers y G. Kaiser (Eds.) Equity in mathematics education: Influences of feminism and culture (pp. 186-199). London: Falmer.

Recibido: 28 de diciembre de 2015

Recepción Modificaciones: 6 de marzo de 2016

Aceptado: 8 de marzo de 2016 